\title{
IMLANNs for Congestion Management in Power System
}

\author{
Nur Zahirah Mohd Ali ${ }^{1}$, Ismail Musirin' ${ }^{2}$, Hasmaini Mohamad ${ }^{3}$, \\ Saiful Izwan Suliman ${ }^{4}$, Hadi Suyono ${ }^{5}$ \\ 1,2,3,4 Faculty of Electrical Engineering, Universiti Teknologi MARA Malaysia, Shah Alam, Selangor, Malaysia \\ ${ }^{5}$ Electrical Engineering Department, Faculty of Engineering, Universitas Brawijaya \\ Jalan MT. Haryono 167 Malang 65145 Indonesia
}

\section{Article Info}

Article history:

Received Apr 1, 2018

Revised Jun 20, 2018

Accepted Jul 7, 2018

Keywords:

IMLANNS

Congested line prediction

FVSI

Voltage collapse

\begin{abstract}
In this paper, Integrated Multi-Layer Artificial Neural Networks (IMLANNs) model has been developed for congested line prediction in a power system. The master characteristic of an ANN is the superiority to achieve complicated input-output mappings through a learning procedure, without exhaustive programming efforts. The IMLANNs model was developed to predict the congested lines in a power system. Before the IMLANNs model is developed, a case study was selected to receive an early result in power system load current during normal condition and contingency based on heavily loaded term. In order to optimize the architecture of the neural network and minimize the computational effort, but those state variables with major impact on the power system are selected as inputs. A pre-developed index, namely Fast Voltage Stability Index (FVSI) is employed as a benchmark to identify the locations declared as congested lines. This indicator was produced which aims for an analytic thinking, sustainable power system when an excessive load was imposed on the power system network. In addition, voltage collapse can be identified when the index is approaching 1.000 or unity. The value of FVSI is chosen as the targeted output in the IMLANNs model. The strength of the proposed IMLANNs model has been validated on the IEEE 30- Bus RTS. Results obtained from the study demonstrated that the proposed IMLANNs is feasible for congested line prediction, which in turns beneficial to power system operators in the planning unit of a utility.
\end{abstract}

Copyright (C) 2018 Institute of Advanced Engineering and Science. All rights reserved.

\section{Corresponding Author:}

Nur Zahirah Mohd Ali, Faculty of Electrical Engineering, Universiti Teknologi MARA Malaysia, Shah Alam, Selangor, Malaysia. Email: nurzahirah_mohdali@yahoo.com

\section{INTRODUCTION}

The need for electricity has led to the increasing burden to power system network due to the rapid growth in most of developing countries. This results the electric power generation sector to experience possible congestion phenomenon. Referable to the extreme overcrowding; a transmission system cannot execute the transfer of force beyond the allowable limits as safety factor needs to be spoken. To overcome this bottleneck, Flexible Alternative Current Transmission Systems (FACTS) devices can be used [1-4]. It helps to cut the extra load on the transmission lines, loss of energy and makes the system more stable. To solve congestion problems, numerous researches have been directed to arrive up with better answers. Many techniques have been developed to identify the exact location of congestion in the transmitting arrangement. Correct congested line identification is important for the installation of FACTS devices. Among the techniques used is voltage stability index, congestion index, flow index performance real power, line utilization factor, sensitivity index and security sensitivity index [1, 3, 5-8]. Consequently, a great deal of 
effort has been devoted to the development of practical tools for analyzing and monitoring voltage stability of power systems using static approaches. The final results from the index will be used as a locator tool that is best for FACTS devices, at once identify the congested bus or line faced in the system.

In this paper, Integrated Multi Layer Artificial Neural Networks (IMLANNs) model has been developed for congested line prediction in choosing power system. The master characteristic of an ANN is the power to achieve complicated input-output mappings through a learning procedure, without exhaustive programming efforts. The IMLANNs model was prepared to predict the FVSI value and congested lines in power system. Before the IMLANNs model is developed, a case study was selected to receive an early result in power system load current during normal condition and contingency based on heavily loaded term. In order to optimize the architecture of the neural network and minimize the computational effort, but those state variables with major impact on the power system are selected as inputs. A pre-developed index, namely Fast Voltage Stabiliy Index (FVSI) is used as a benchmark to identify the locations declared as congested lines [9]. This index was developed by Ismail Musirin et. al which aims for an analysis, sustainable power system when an excessive load was imposed on the power system network. In addition, voltage collapse can be identified when the index is approaching 1.000 or unity. The value of FVSI is chosen as the targeted output in the IMLANNs model. The effectiveness of the proposed IMLANNs model has been tested on the IEEE 30-Bus RTS.

\section{RESEARCH METHOD}

\subsection{Congested Lines Identification Technique}

In general, electric power system delivers three major ingredients in order to supply, transmit and utilize the power to the consumer. As power system can operate under stress conditions, the ability to maintain voltage stability becomes a growing business organisation to the electrical engineer. In parliamentary law to predict the voltage stability, the development of accurate methods is important in preventing such conditions. It is also important in the interest of power system planning and arrangement. Congestion problem is likewise linked up to the matters above. It was mainly due to reactive power variation. A set of electrical loads are in the shape of heavy machines confining windings, transformers and other factors that have reactive power effect. The expansion in a reactive power request yet poorly in reactive power support, has known a power system to be in an anxiety condition. These events have subsequently motivated further research in the area of congestion analysis. Many techniques have been developed in order to alleviate the congestion in a power system [10-19]. The result from voltage stability analysis may lead to weak bus identification or critical line determination.

Firstly, it is fundamental to know what kind of line-based voltage dependability record that is worthy to be adopted. In this paper, the Fast Voltage Stability Index (FVSI) has been decided for giving the dependable positioning rundown to a power system administrator. FVSI was broadly actualized as a rule concerning voltage stability and checking because of its reliance on reactive power rather than real power. Being proposed by I. Musirin [20], dependability of FVSI in voltage stability appraisal has been legitimized in $[21,22]$, where the record was connected for weak cluster identification, most extreme loadability and reactive power planning. In different cases, the $\mathrm{Q}-\mathrm{V}$ curve is used for monitoring load margin between operating point and voltage collapse point. By virtue of that, FVSI turns into an ideal line-based list; clarifying the motivation behind why it has been picked as the record to be followed by means of the proposed multi-model ANN algorithm. FVSI of l-th line is given in (1)

$$
F V S I=\frac{4 Z_{l}^{2} Q_{r}}{V_{s}^{2} X_{l}}
$$

where $Z_{l}$ is the line impedance, $X_{l}$ is the line reactance, $V_{s}$ is the sending end voltage and $Q_{r}$ is the less receiving end power. It must to be noticed that for a regular power system that is free from voltage breakdown risk, FVSI must be not as much as unity.

\section{1) Maximum Permissible Load}

Voltage in power system can become unstable when the load on the reactive power increases and exceeds the maximum level. To get an idea of the maximum load on the stagecoach before the potential is not static, the voltage stability assessment is done using pre- developed FVSI. Maximum permissible load or also termed as maximum loadability is an important detail to indicate power system strength. Maximum loadability determines whether a power system network is in its secure area or vice versa. The high value of maximum loadability allows to system to add more load to a particular load bus. On the other hand, low maximum loadability value only allows small load to be carried on to the corresponding load bus. 
Nevertheless, $\mathrm{Q}_{\mathrm{d}}$-FVSI can be utilised to indicate the point of $\mathrm{Q}_{\mathrm{dmax}}$ for a chosen load in a transmission system. Algorithm for maximum loadability identification is given as follows:

i. Select the load bus.

ii. Set the loading condition at the particular load bus.

iii. Run the load flow program using the Newton - Raphson method for the base case.

iv. Gradually increase the reactive power loading at the chosen load bus until the FVSI value close to 1.000 .

v. Extract the line with the FVSI value closed in 1.000, this line called as the congested line with respect to a bus.

vi. Rank the top 5 lines with high FVSI values.

vii. Choose other load bus and repeat step (i) to (vi).

viii. Identify $\mathrm{Q}_{\mathrm{dmax}}$ for every load bus.

\section{2) Identification of Congested Lines}

While power system is affected by various disturbances such as outages and the increment of load, voltage security can be possibly experienced by the system. It is related to the continous of a maximum active or reactive power through the transmission line which is transferred from the generation centre to the load. For example, when consumer used a high air conditioning load for a long time in a hot summer day, voltage will drop in one or several areas. It has been recognized that, electrical power equipment such as transformer taps and generators have their operating limits for preventing the system from receiving voltage collapse. A generator or transmission line may possibly experience tripping which can be ascribable to an unexpected incident. Apparently, the local reactive power support is demanded for the reactive power cannot be transported due to high losses. The voltage may drop speedily and can lead to voltage collapse of the whole system.

In this paper, pre-developed FVSI was used to find out the congested line when the maximum loading is injected to the system. A load bus in a power system is typically associated with a few lines forming an interconnected system in a power system. The increment in reactive power loading at a specific loaded bus would specifically raise the FVSI values on the lines associated to it. (The FVSI values on different lines in the power system might likewise increment, however the line with the highest FVSI quality is ordinarily associated specifically with the loaded bus). Figure 1 shows the profile of FVSI versus reactive power loading for congested line identification. FVSI increases steadily when the reactive loading is increased. For instance, a FVSI value at line $1\left(\mathrm{~L}_{1}\right)$ continue to increase until it reaches the maximum permissible load, $Q_{\operatorname{dmax}}$. The FVSI value at this maximum permissible load is within 0.9 1.0; which falls below the congested region. Similar scenario can be observed for $L_{2}$ and $L_{3}$; where FVSI values increase accordingly as $Q_{d}$ is increased. Nevertheless, $L_{2}$ and $L_{3}$ do not enter the congested region even $Q_{d}$ has reached its $\mathrm{Q}_{\mathrm{dmax}}$ point since the corresponding values have not reached 0.9 to 1.0 region. It implies that $\mathrm{L}_{2}$ and $\mathrm{L}_{3}$ are all the same inside the stable region.

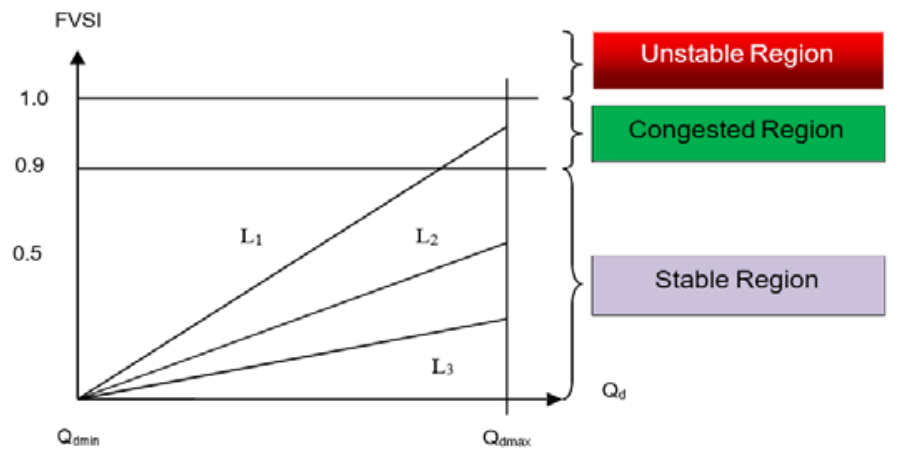

Figure 1. FVSI versus reactive power loading for congested line identification

\subsection{Proposed Integrated Multilayer Artificial Neural Networks (IMLANNs)}

This paper proposes model Integrated Multilayer Artificial Neural Networks (IMLANNs) as the technique to predict index values for congested line. The IMLANNs models has been trained by utilizing of two hidden layer feedforward network. During the training process, an input- output pair is chosen from the 
set of training data, while the weights and biases of the network are randomized. In order to calculate the actual output, the selected input-output pair is applied to the IMLANNs. Consequently, the error of the prediction process can be determined by calculating the difference between the actual output and the targeted output. The error can be minimized by adjusting the weights and biases of the network according to the specific learning rules used by the selected learning algorithm. In this model, the training and testing processes have been integrated together. This is expected to reduce the computational burden experienced in the traditional approach.

Over the year, ANN has been used extensively in various fields of power engineering especially for solving voltage stability analysis and so on [23,24]. In this paper, the Integrated Multi-Layer Artificial Neural Networks (IMLANNs) with Levenberg-Marquardt backpropagation training algorithm is employed for predicting purposes.

\subsection{Identification of Congested Lines} explained:

This section presents the whole procedures of the proposed IMLANNs model. The process is

i. $\quad$ Choose the load bus. In IEEE 30-Bus, there are 11 load buses that have been chosen for the testing process.

ii. $\quad$ Read system data: IEEE 30-Bus RTS.

iii. Set the loading condition. This stage allows the system to run at the maximum permissible load.

iv. Run load flow.

v. Calculate FVSI values for all lines and identify the congested lines.

vi. Build the raw data patterns for the ANN. If the number of patterns is not adequate, adjust the loading condition.

vii. Cluster the training patterns and testing patterns.

viii. Call the training data patterns.

ix. Initialize the training parameters. In the stage of training process, the numbers of nodes in each hidden layer, momentum rate, learning rate and other training parameters have been conducted by extensive experiments in order to determine the optimal value.

x. Develop the training configuration. The number of neurons in the first hidden layer, the number of neurons in the second hidden layer, the type of active of activation functions, number of iterative updates (epochs) and the mean square error goal can be varied in the process of determination optimal types or values.

xi. Perform the training data patterns.

xii. If the solution does not converge, vary the training parameters.

xiii. $\quad$ Save the IMLANNs model.

xiv. Call the testing data patterns. Retreive the saved IMLANNs model. The testing process is conducted consecutively after obtaining the best trained ANN model.

xv. Perform testing process. Calculate the absolute error $\left(E_{a b s}\right)$ as follows:

$E_{a b s}=\left|o_{c}-o_{p}\right|$

where $\mathrm{O}_{c}$ is the calculated output and $\mathrm{O}_{\mathrm{p}}$ is the predicted output. $n$ is the number of training patterns involved. The regression analysis is used to minimize the prediction error when fitting the predicted output to the targeted output.

xvi. If $\mathrm{R}$ is not close to 1.00 , repeat step xii.

xvii. Tabulate the IMLANNs output and targeted output.

\section{RESULTS AND ANALYSIS}

This section is divided to three main subsections, which are maximum permissible load, congested lines identification and proposed IMLANNs model for congested line identification. All the coding tasks were implemented via MATLAB software and have been utilised in IEEE 30-Bus RTS.

\subsection{Maximum Permissible Load}

As described in the earlier sections, maximum permissible load was estimated using the predeveloped FVSI. In this study, FVSI was implemented as the indicator to estimate the maximum loadability and to identify the congested line in the system. Maximum permissible load is estimated by increasing the reactive loading at the chosen load buses. The maximum permissible load was identified as the reactive 
loading at which the FVSI values reaches 0.9. Table 1 tabulates the ranges for 11 load buses in IEEE 30-Bus RTS. Load at each loaded bus was increased by 10MVAR within the ranges tabulated in the table.

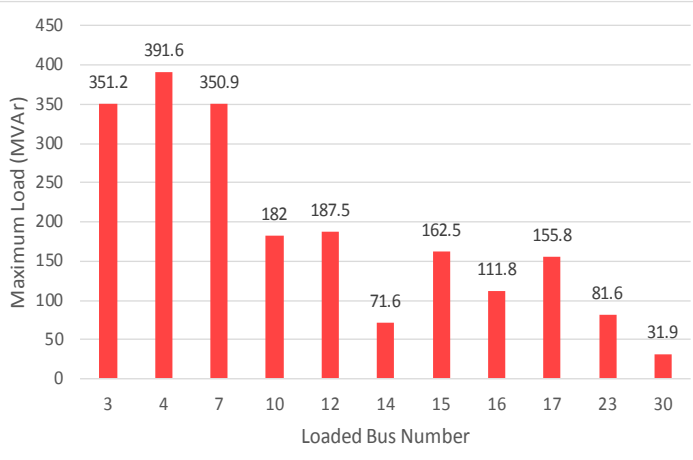

Figure 2. Maximum permissible load in IEEE 30Bus RTS
Table 1. Load Ranges for 11 Loaded Buses in IEEE 30-Bus RTS

\begin{tabular}{ccc}
\hline Load Bus & $\begin{array}{c}\text { IEEE } \\
\text { 30-Bus RTS } \\
\mathrm{Q}_{\mathrm{dmin}}\end{array}$ & $\mathrm{Q}_{\mathrm{dmax}}$ \\
\hline 3 & 1.2 & 351.2 \\
4 & 1.6 & 391.6 \\
7 & 10.9 & 350.9 \\
10 & 2.0 & 182.0 \\
12 & 7.5 & 187.5 \\
14 & 1.6 & 71.6 \\
15 & 2.5 & 162.5 \\
16 & 1.8 & 111.8 \\
17 & 5.8 & 155.8 \\
23 & 1.6 & 81.6 \\
30 & 1.9 & 31.9 \\
\hline
\end{tabular}

The graphical representation indicating the maximum permissible load in IEEE 30-Bus RTS is depicted in Figure 1. The maximum permissible load for buses 3, 4, 7, 12, 14, 15, 16, 17, 23 and 30 are shown in the figure. The permissible load for bus 4 is the highest among others with its corresponding value of 391.6MVar. The second highest permissible load is experienced at bus 3 with its value of 351.2MVar. Bus 30 shows the lowest permissible load with its value shown in the figure. All the maximum permissible load for the chosen loaded buses are determined in accordance to the limitation of FVSI. The permissible load for each load bus in the system is different. This is due to the preinstalled capacity determined by the utility. Low permissible load would lead to low amount of load that can be subjected to the load bus. This can also imply a weak bus.

On the other hand, high permissible load value implies a strong bus. This will allow the utility to connect higher load value at this bus. On overall, maximum permissible load would be very beneficial to the power system operator or planner in managing their transmission system capacity. Offline studies on system capacity are normally conducted to allow future planning and monitor the system capacity. Thus future load increment forecasted by the utility can be based on this information.

\subsection{Congested Lines Identification}

FVSI is used as the device to determine the congested lines the system. The line with the FVSI index value close to 1.000 indicates how close the particular line to its instability point. Hence the congested line can be identified in order to manage the congestion condition in the system with the installation of FACTS devices. Congested line identification was conducted on IEEE 30-Bus RTS. This is significant for the data generation in the proposed IMLANNs model. The results of congested lines identification for preIMLANNs maximum permissible load in IEEE 30-Bus RTS are tabulated in Table 2.

From the first column, 5 loaded buses with the highest maximum permissible load are chosen to identify their congested lines. Bus 3 with the highest maximum permissible load of 351.2MVar exhibited the highest corresponding FVSI value of 0.9568. The top 5 FVSI values recorded for this case. Bus 3 was loaded can be seen in the table. Line 4 is identified as the congested line for this case, which connects bus 3 and bus 4 . The 5 lines with the top FVSI values are also shown in the table. At bus 4, with the maximum permissible load of 391.6MVar, line 15 connecting bus 4 and bus 12 is determined as the congested line with its FVSI value of 0.9808 . The congested line when bus 7 was loaded is line 8 with its FVSI value of 0.9757. Congested lines for other cases can be referred to the same table. 
Table 2. Congested Line Identification at Maximum Permissible Load in IEEE 30-Bus RTS

\begin{tabular}{|c|c|c|c|c|c|}
\hline $\begin{array}{c}\text { Loaded } \\
\text { Buses }\end{array}$ & $\mathrm{Q}_{\mathrm{dmax}}$ & $\begin{array}{c}5 \text { Highest } \\
\text { FVSI }\end{array}$ & $\begin{array}{c}\text { Congested } \\
\text { Line } \\
\end{array}$ & $\begin{array}{c}\text { From } \\
\text { Bus }\end{array}$ & $\begin{array}{c}\text { To } \\
\text { Bus } \\
\end{array}$ \\
\hline \multirow{5}{*}{3} & \multirow{5}{*}{351.2} & 0.9568 & 4 & 3 & 4 \\
\hline & & 0.8448 & 15 & 4 & 12 \\
\hline & & 0.8211 & 2 & 1 & 3 \\
\hline & & 0.5731 & 7 & 4 & 6 \\
\hline & & 0.4839 & 3 & 2 & 4 \\
\hline \multirow{5}{*}{4} & \multirow{5}{*}{391.6} & 0.9808 & 15 & 4 & 12 \\
\hline & & 0.7020 & 7 & 4 & 6 \\
\hline & & 0.5829 & 3 & 2 & 4 \\
\hline & & 0.5356 & 2 & 1 & 3 \\
\hline & & 0.4437 & 13 & 9 & 11 \\
\hline \multirow{5}{*}{7} & \multirow{5}{*}{350.9} & 0.9757 & 8 & 5 & 7 \\
\hline & & 0.8020 & 9 & 6 & 7 \\
\hline & & 0.4403 & 11 & 6 & 9 \\
\hline & & 0.4169 & 12 & 6 & 10 \\
\hline & & 0.3757 & 15 & 4 & 12 \\
\hline \multirow{5}{*}{10} & \multirow{5}{*}{182.0} & 0.9594 & 13 & 9 & 11 \\
\hline & & 0.5886 & 12 & 6 & 10 \\
\hline & & 0.4891 & 14 & 9 & 10 \\
\hline & & 0.3540 & 16 & 12 & 13 \\
\hline & & 0.3286 & 19 & 12 & 16 \\
\hline \multirow{5}{*}{12} & \multirow{5}{*}{187.5} & 0.9195 & 16 & 12 & 13 \\
\hline & & 0.3693 & 15 & 4 & 12 \\
\hline & & 0.3670 & 13 & 9 & 11 \\
\hline & & 0.2337 & 19 & 12 & 16 \\
\hline & & 0.2325 & 21 & 16 & 17 \\
\hline
\end{tabular}

Table 3. IMLANNs Model 2 for Congested Line Identification with Maximum Permissible Load in the 30-IEEE Bus RTS

\begin{tabular}{|c|c|c|c|}
\hline \multirow[b]{2}{*}{$\begin{array}{c}\text { Loaded } \\
\text { Buses }\end{array}$} & \multicolumn{2}{|c|}{ Output } & \multirow[b]{2}{*}{ Error $_{a b s}$} \\
\hline & $\begin{array}{c}\text { Congested Line - } \\
\text { NR }\end{array}$ & $\begin{array}{l}\text { Congested Line - } \\
\text { IMLANNs }\end{array}$ & \\
\hline 3 & 4 & 3.9999 & 0.0001 \\
\hline 4 & 15 & 14.9999 & 0.0001 \\
\hline 7 & 8 & 7.9999 & 0.0001 \\
\hline 10 & 13 & 13.0121 & 0.0121 \\
\hline 11 & 16 & 16.0111 & 0.0111 \\
\hline
\end{tabular}

Table 4. Regression Performance at Maximum Permissible Load in IEEE 30-Bus RTS

\begin{tabular}{cccccc}
\hline Performance Type & Bus 3 & Bus 4 & Bus 7 & Bus 10 & Bus 12 \\
\hline R for Training & 0.99999 & 0.99999 & 0.99999 & 0.99396 & 0.96789 \\
R for Testing & 1.00000 & 1.00000 & 1.00000 & 0.99283 & 0.96789 \\
\hline
\end{tabular}

\subsection{IMLANNs Model for Congested Line Identification at Maximum Permissible Load}

The proposed IMLANNs model has been tested on IEEE 30-Bus RTS. This is meant for congested line identification at maximum permissible load. The proposed IMLANNs model consists of 5 inputs as discussed in the previous sections are the control variables used to predict the congested lines. The results for predicted congested line using IMLANNs at maximum permissible loading are tabulated in Table 3.

In this paper, 5 loaded buses were chosen from the 11 loaded buses extracted from Figure 2. The values for the corresponding maximum permissible loads are indicated in the figure. The results of congested line predicted using IMLANNs were compared with the respect to the Congested Line-NR (targeted) calculated by Newton Raphson load flow study. The last column indicates the absolute errors resulted from the study. Results obtained from the study show that the proposed IMLANNs managed to predict accurately when compared with the targeted congested lines. For instance, when the system is subjected to load at buses 3 and 10; the predicted congested lines give values at 3.9999 and 13.0121. This implies that the predicted congested lines are actually lines 4 ( IMLANNs output $=3.9999)$ and 13 (IMLANNs output = 13.0121). Apparently, the accuracy is significantly high indicated by their low absolute errors appeared in the last column. The accuracy values for other load buses are consistenly low. This signifies the accuracy of the developed ANN model.

The regression analysis performance for this model was consequently evaluated. This process was conducted during testing process. The testing process was conducte consecutively after obtaining the best trained IMLANNs model. The regression coefficient $(\mathrm{R})$ are computed to qualify the prediction performance of IMLANNs. The value of $\mathrm{R}$ is determined using the neural network toolbox in MATLAB. In Table 4, the results of regression analysis performance for this model was recorded. From this table, this model experiences significantly $\mathrm{R}$ values during training and testing processes. For example, when Bus 3 was tested, the $\mathrm{R}$ value is greater than 0.90000 while its value 1.0000 during testing. The same scenario can be observed for other loaded buses. This implies that this modell performed well when implemented in the IEEE 30-Bus RTS. 


\section{CONCLUSION}

This paper has presented study on Intergrated Multi-Layer Artificial Neural Network (IMLANNs) for congested line identifications. In this study, one model IMLANNs has been developed to perform congested line at maximum permissible load. Test was conducted on the reliability test systems namely IEEE 30-Bus RTS. Training and testing processes were conducted independently one at a time for all cases because the developed IMLANNs network is unique to address each problem. Based on the observed result for the test system it can be concluded that the proposed IMLANNs technique managed to successfully predict congested line under this scenario. The results from this study will be used for the next study.

\section{ACKNOWLEDGEMENT}

The authors would like to acknowledge the Institute of Research Management and Innovation (IRMI) UiTM Shah Alam, Selangor, Malaysia for the financial support of this research. This research is supported by IRMI under the BESTARI Research Grant Scheme with project code: 600-IRMI/MyRA 5/3/BESTARI (0026/2017).

\section{REFERENCES}

[1] Singh SN, David AK. Optimal Location of FACTS Devices for Congestion Management. Electric Power Systems Research. 2001; 58(2): 71-79.

[2] Karatekin CZ, Uçak C. Sensitivity Analysis Based on Transmission Line Susceptances For Congestion Management. Electric Power Systems Research. 2008; 78(9): 1485-1493.

[3] Mandala M, Gupta CP. Optimal placement of TCSC for transmission congestion management using hybrid optimization approach. International Conference on IT Convergence and Security (ICITCS). 2013;1-5.

[4] Sanjari MJ, Fathi H, Gharehpetian GB, Tavakoli A. HSA-based optimal placement of shunt FACTS devices in the smart grid considering voltage stability. Iranian Conference on Smart Grids (ICSG). 2012; 2: 1-6.

[5] Mahdad B, Srairi K. Security Optimal Power Flow Considering Loading Margin Stability using Hybrid FFAPS Assisted with Brainstorming Rules. Applied Soft Computing. 2015; 35: 291-309.

[6] Shih Chieh H, Chien Chih C, Hsin Min W. Congestion cost allocation and congestion indices for a competitive electricity market. IEEE International Conference on Industrial Technology (ICEEI). 2002; 2: 854-859.

[7] Sawan S, Chanda S, Sengupta S, Chakrabarti A, De A. Alleviation of line congestion using multiobjective particle swarm optimization. International Conference on Electrical Engineering and Informatics (ICEEI). 2011; $1-5$.

[8] Smt. Ushasurendra SSP, Congestion Management in Deregulated Power Sector using Fuzzy Based Optimal Location Technique for Series Flexible Alternative Current Transmission System (FACTS) Device. Journal of Electrical and Electronics Engineering Research. 2012; 4: 12-20.

[9] Musirin I, Rahman TKA. Novel fast voltage stability index (FVSI) for voltage stability analysis in power transmission system. Student Conference on Research and Development (SCOReD) 2002; 265-268.

[10] Tabandeh A, Abdollahi A, Rashidinejad M. Security-based congestion management considering power system components uncertainty. 21st Conference on Electrical Power Distribution Networks Conference (EPDC). 2016; 172-179.

[11] Hosseinipoor NA, Nabavi SMH. Social welfare maximization by optimal locating and sizing of TCSC for congestion management in deregulated power markets. International Conference on Power System Technology (POWERCON). 2010; 1-5.

[12] Hosseini SA, Amjady N, Shafie-khah M, Catalão JPS.A New Multi-Objective Solution Approach to Solve Transmission Congestion Management Problem of Energy Markets. Applied Energy, 2016; 165: 462-471.

[13] Hashemzadeh H, Hosseini SH. Locating series FACTS devices using line outage sensitivity factors and particle swarm optimization for congestion management. IEEE Power and Energy Society General Meeting (PES09). 2009; 1-6.

[14] Guguloth R, Kumar TKS, LMP calculation and OPF based congestion management in deregulated power systems. ELEKTRO, 2016; 299-304. 